Gynecologic and

Obstetric Investigation
Gynecol Obstet Invest 2010;70:40-46

DOI: $\underline{10.1159 / 000284949}$
Received: July 27, 2009

Accepted after revision: November 23, 2009

Published online: February 17, 2010

\title{
Interrelationship of Cytokines, Hypothalamic-Pituitary-Adrenal Axis Hormones, and Psychosocial Variables in the Prediction of Preterm Birth
}

\author{
B.D. Pearce $^{a} \quad$ J. Grove $\quad$ E.A. Bonney ${ }^{d} \quad$ N. Bliwise $^{b} \quad$ D.J.Dudley ${ }^{e} \quad$ D.E. Schendel ${ }^{f}$ \\ P. Thorsen ${ }^{9}$ \\ ${ }^{a}$ Department of Epidemiology, Rollins School of Public Health, and ${ }^{b}$ Department of Psychology, Emory University, \\ Atlanta, Ga., USA; ' Department of Human Genetics and Bioinformatics, Research Center, Institute of Public Health, \\ University of Aarhus, Denmark; ${ }^{d}$ Department of Obstetrics and Gynecology, University of Vermont, Burlington, \\ Vt., ${ }^{\text {eDepartment }}$ of Obstetrics and Gynecology, University of Texas Health Science Center, San Antonio, Tex., \\ and ${ }^{\mathrm{f} C e n t e r s ~ f o r ~ D i s e a s e ~ C o n t r o l ~ a n d ~ P r e v e n t i o n ~(N C B D D D), ~ A t l a n t a, ~ G a ., ~ a n d ~}{ }^{9}$ Department of Epidemiology and \\ Biostatistics, School of Public Health, Drexel University, Philadelphia, Pa., USA
}

\section{Key Words}

Stress • Corticotropin-releasing hormone • Inflammation •

Neuroendocrine $\cdot$ Interleukin- 6

\begin{abstract}
Background/Aims: To examine the relationship of biological mediators (cytokines, stress hormones), psychosocial, obstetric history, and demographic factors in the early prediction of preterm birth (PTB) using a comprehensive logistic regression model incorporating diverse risk factors. Methods: In this prospective case-control study, maternal serum biomarkers were quantified at 9-23 weeks' gestation in 60 women delivering at $<37$ weeks compared to 123 women delivering at term. Biomarker data were combined with maternal sociodemographic factors and stress data into regression models encompassing 22 preterm risk factors and 1storder interactions. Results: Among individual biomarkers, we found that macrophage migration inhibitory factor (MIF), interleukin-10, C-reactive protein (CRP), and tumor necrosis factor- $\alpha$ were statistically significant predictors of PTB at all cutoff levels tested (75th, 85th, and 90th percentiles). We fit multifactor models for PTB prediction at each biomarker cut-
\end{abstract}

off. Our best models revealed that MIF, CRP, risk-taking behavior, and low educational attainment were consistent predictors of PTB at all biomarker cutoffs. The 75th percentile cutoff yielded the best predicting model with an area under the ROC curve of 0.808 ( $95 \% \mathrm{Cl} 0.743-0.874)$. Conclusion: Our comprehensive models highlight the prominence of behavioral risk factors for PTB and point to MIF as a possible psychobiological mediator.

Copyright $\odot 2010$ S. Karger AG, Basel

\section{Introduction}

Preterm birth (PTB, <37 weeks' gestation) has multiple etiologies and contributing factors [1-3]. In addition to physiological risk factors such as infections and cervical insufficiency [2], there are psychosocial antecedents and causative pathways thought to involve maternal stress and depression [3].

The paucity of reliable biomarkers for PTB that can be obtained by noninvasive means early in pregnancy makes PTB one of the most challenging conditions for obstetricians worldwide. One approach to delineating useful bio-

\section{KARGER}

Fax +41613061234 E-Mail karger@karger.ch www.karger.com
(C) 2010 S. Karger AG, Basel

0378-7346/10/0701-0040\$26.00/0

Accessible online at:

www.karger.com/goi
Brad D. Pearce, $\mathrm{PhD}$

1518-002-4AA (RSPH: Epidemiology)

Grace C. Rollins Bldg

Emory University, Rollins School of Public Health, Atlanta, GA 30322 (USA)

Tel. +1 404727 4914, Fax +1 404727 8737, E-Mail bpearce@ emory.edu 
markers is to focus on potential mechanisms or groups of related mechanisms. Many investigators have shown that acute phase reactants such as C-reactive protein (CRP), and proinflammatory cytokines like IL- 6 and tumor necrosis factor- $\alpha$ (TNF- $\alpha$ ), are potential markers for the infection/inflammation-mediated PTB pathways [4-9]. Recently there has been focus on mechanisms that regulate this process, including IL-10 [7].

Another regulator of inflammation, macrophage migration inhibitory factor (MIF), was reported by our groups to be elevated in maternal serum months prior to preterm delivery [10]. The mechanism linking MIF with PTB has not been discerned. In humans, MIF is expressed in multiple tissues including immune cells, placenta, and fetal membranes [11]. MIF is generally proinflammatory and counteracts the immunosuppressive activity of glucocorticoids [12]. Thus MIF is believed to be an important determinant of the balance between inflammatory and antiinflammatory processes, but there are limited data on MIF regulation in human pregnancy $[10,11,13]$. MIF has properties of a stress hormone as indicated by its expression in the brain, its high concentration of secretory granules in the anterior pituitary, and its release by corticotropin-releasing hormone $(\mathrm{CRH})$ in vitro and by psychological stress in rats $[12,14]$. Thus, MIF is well positioned to function as a behaviorally active hormone and neuroendocrine modulator in pregnancy.

The goal of the current study was to develop a predictive model of PTB that incorporates MIF and other biological and socio-behavioral risk factors that can be assessed early in pregnancy.

\section{Materials and Methods}

Our prior papers have described subject characteristics for this case-control study nested in a prospective cohort of women receiving prenatal care at the Department of Obstetrics and Gynecology at Odense University Hospital, Denmark. Briefly, of 2,927 women who completed all test and questionnaire requirements, 170 delivered preterm, which was defined as $<37$ full gestational weeks ( $<259$ days of gestation), and 60 of these met the final inclusion criteria for idiopathic preterm delivery. As described previously for this population [1], ultrasonographic measurements of the biparietal diameter and the femur length of the fetus at the 18th week of gestation confirmed gestational age and the estimated date of delivery for $97.5 \%$ of the participants. Idiopathic PTB was defined as PTB without a cause that could be indentified from clinical findings or clinical diagnostic laboratory measurements performed during pregnancy or at delivery. Thus the PTB group consisted of 60 women with idiopathic PTB as described previously $[1,10]$. A subset of women in the cohort who delivered at term (261-303 days of gestation) served as the term-delivery comparison group (controls) as described previously $[1,10]$. Both groups of women had blood samples taken before 24 weeks' gestation, allowing case-control comparison for early blood biomarkers of subsequent delivery status. Accordingly, this sample was taken before it was known which women would deliver preterm.

All participants gave written informed consent that met the requirements of the scientific ethics committee for Vejle and $\mathrm{Fu}$ nen Counties, Denmark, and the institutional review board for research on human subjects, Centers for Disease Control and Prevention (Atlanta, Ga., USA). Data collection methods were approved by the Danish Data Protection Agency. A pelvic examination including clinical observations was performed on each participant upon enrollment as described previously for this cohort [1]. Estimates of gestational age and estimated date of delivery were calculated from the date of the last menstrual period.

The obstetrical medical record was supplemented with questionnaire data concerning maternal behaviors (e.g. smoking, seat belt usage), psychosocial stress, socioeconomic factors, work demands, and prior obstetrical problems [1]. Socioeconomic status indices were derived from detailed job descriptions and educational backgrounds for each participant and her partner as described previously [1].

In choosing factors to include in predictive models, we selected variables that can be readily determined at the first routine prenatal visit. Thus, in addition to serum biochemical markers, the predictive models included maternal demographic, historical, and psychosocial factors that were selected based on reported association with PTB in the literature $[1,2]$ and sufficient frequency in our sample (e.g. non-Caucasian race was not included as a factor because it was rare in our sample). The relationship of obesity and PTB risk has not been resolved, but maternal obesity is positively correlated with proinflammatory markers and hence we included pre-pregnancy BMI $>30$ (dichotomized) in our models [15].

\section{Serum Analysis}

Serum cytokines (IL-1 $\beta$, IL- 6 and TNF- $\alpha$ ) were assayed by sandwich ELISA according to the manufactures' protocol (R \& D Systems, Minneapolis, Minn., USA) after a single thaw. For IL-10, serum samples were analyzed after two separate thaws, also using an R \& D Systems kit. Levels of MIF, CRH (free and bound), cortisol, and CRP were measured by enzyme-linked immunoassays, and have been reported in prior publications $[1,4,10]$. While the coefficients of variation (CVs) for the other cytokines were $<10 \%$, the MIF inter-assay CV is somewhat high (17.6\%). However, each MIF assay run included both cases and control samples distributed on the plate, with the assay technician blinded to case-control status.

\section{Statistics}

The data were analyzed as a nested case-control study [10]. Because the biomarker measurements were not normally distributed even after a natural log transformation, data were analyzed using non-parametric methods (Spearman rank order correlation) or were dichotomized and analyzed using logistic regression. Odds ratios with $95 \%$ confidence intervals (CIs) were used to assess effect size.

As a precursor to building a predictive model for PTB, all biomarkers were dichotomized at the 75 th, 85 th and 90 th percentiles 
based on values for women who delivered at term, except for free (unbound) $\mathrm{CRH}$, which had a high number of measurements below the detection limit. Free $\mathrm{CRH}$ was dichotomized at the lower limit of detection.

Using these three different cutoff levels to dichotomize molecular biomarkers, we estimated logistic regression models of 2-7 terms encompassing 22 risk factors and their first-order interactions. Based on pilot studies of model stability, we reasoned that the data would be able to fit 6 terms and therefore chose to run models with up to 7 terms to test this assumption. The risk factors entered into models were: MIF, TNF, IL-6, IL-10, CRP, total $\mathrm{CRH}$, bound $\mathrm{CRH}$, free $\mathrm{CRH}$, cortisol, tobacco smoking (10 or more per day), previous PTB, lack of basic education, mothers SES, serious maternal medical condition, nullipara, BMI $>30$, standing at work more than $6 \mathrm{~h} /$ day, self-reported social stress, stressful life events, risk-taking behavior, previous pelvic inflammatory disease, and bacterial vaginosis at 1st prenatal visit.

Predictive models for PTB outcome were built by estimating all possible logistic regression models from the aforementioned measured variables including first-order interactions. The best predictive models were selected based on the highest areas under the receiver operating characteristic (ROC) curves (and 95\% CIs) for PTB outcome. Since the concentration of free $\mathrm{CRH}$ was below the detection limit of the assay in $91 \%$ of the controls, it was decided to use the lowest detected concentration value for dichotomization throughout all logistic regression analyzes.

Analyses were carried out using Stata 10 (StataCorp LP, College Station, Tex., USA).

\section{Results}

Characteristics of subjects selected for this case-control study are shown in table 1 , which are consistent with our prior reports on this cohort $[1,10]$.

We examined the relationship between the concentration of cytokines and hormones at 9-23 weeks' gestation and PTB outcome (table 2). Elevation of MIF, TNF, IL-10 and CRP showed a statistically significant association with subsequent PTB at all three cutoff levels, whereas IL-6 and cortisol reached statistical significance only at the 85th and 90th percentile cutoff, and for bound and total CRH statistical significance was only at the 75th and 85th percentile cutoffs. High levels of free CRH failed to reach significance with $\mathrm{OR}=2.04,95 \%$ CI 0.81-5.10.

Next we examined the role of these biomarkers and diverse PTB risk factors in comprehensive predictive models. Using the three different cutoff levels for the molecular biomarkers, we estimated logistic regression models of 2-7 terms encompassing 22 risk factors and their first-order interactions as described in the methods section.

Model stability began to deteriorate at 7 factors so we limited our search of the best predicting model to those
Table 1. Characteristics of the women

\begin{tabular}{lcc}
\hline Patient characteristic & Term & Preterm \\
\hline Number & 123 & 60 \\
Previous preterm delivery* & $6(4.9 \%)$ & $11(18.3 \%)$ \\
Nulliparity & $61(49.6 \%)$ & $30(50.0 \%)$ \\
Smoking & $12(9.8 \%)$ & $4(6.7 \%)$ \\
BMI >30 & $6(4.9 \%)$ & $5(8.3 \%)$ \\
Serious medical disease & $5(4.1 \%)$ & $5(8.3 \%)$ \\
Previous PID & $29(23.6 \%)$ & $14(23.3 \%)$ \\
BV at first visit & $23(18.7 \%)$ & $9(15.0 \%)$ \\
Maternal age at delivery ${ }^{1, *}$ & $29.2(20.0,40.1)$ & $27.4(18.7,41.9)$ \\
Mother lacks basic educa- & $3(2.4 \%)$ & $6(10.0 \%)$ \\
tion & & \\
Low maternal SES & $21(17.1 \%)$ & $12(20.0 \%)$ \\
Risk-taking behavior* & $6(4.9 \%)$ & $9(15.0 \%)$ \\
Social stress & $13(10.6 \%)$ & $10(16.7 \%)$ \\
Major stressful life events & $4(3.3 \%)$ & $3(5.0 \%)$ \\
Standing at work & $57(46.3 \%)$ & $33(55.0 \%)$ \\
GA at first visit, weeks ${ }^{1, *}$ & $16.0(9.0,23.0)$ & $18.4(9.3,21.9)$ \\
GA at delivery, weeks ${ }^{1 *}$ & $40.4(37.3,43.3)$ & $35.8(29.9,36.9)$ \\
Birth weight, ${ }^{2, *}$ & $3,574(573)$ & $2,632(492)$
\end{tabular}

Percentages are within outcome category. Smoking $=\geq 10$ cigarettes/day; $\mathrm{BMI}=$ body mass index; PID = pelvic inflammatory disease; $\mathrm{BV}=$ bacterial vaginosis; $\mathrm{GA}=$ gestational age; $\mathrm{SES}=$ socioeconomic status; standing at work: $>6$ h/day. Risk-taking behavior was indicated by a lack of seat-belt usage, and social stress and stressful life events by self-report.

${ }^{*} \mathrm{p}<0.05$ for case-control comparison (tests comparing medians were continuity corrected).

${ }^{1}$ Median (range). ${ }^{2}$ Mean (standard deviation).

with a maximum of 6 terms including interaction terms. We ranked the models based on the lower limit of the $95 \%$ CI for the ROC curve areas and selected the highest ranked model at each biomarker cutoff as our best predicting model (table 3). MIF was consistently included in all of the top ranking models regardless of the number of terms in the model. Selecting the top 5\% of models with 6 predictors, MIF was in $99 \%$ of those using the 75 th percentile cutoff, $92 \%$ of those using the 85 th percentile cutoff, and 55\% of those using the 90th percentile cutoff. Figure 1 shows a comparison of the ROC curves for the best model (derived from 75\% cutoff) versus MIF alone.

To gain a better understanding of possible regulatory pathways for MIF, we determined whether MIF was correlated with levels of other cytokines in these serum samples (table 4). MIF was positively correlated with TNF but not IL-6 or IL-10. The correlation coefficient for TNF was similar for women with term delivery $\left(\mathrm{r}_{\mathrm{s}}, 0.221\right)$ and preterm delivery $\left(r_{s}, 0.215\right)$, though the latter did not reach 
Table 2. Association of individual biomarkers with subsequent preterm delivery

\begin{tabular}{|c|c|c|c|c|c|c|c|c|c|}
\hline \multirow[t]{3}{*}{ Biomarker } & \multicolumn{9}{|l|}{ Test cutoff } \\
\hline & \multicolumn{3}{|c|}{ 75th percentile } & \multicolumn{3}{|c|}{ 85th percentile } & \multicolumn{3}{|c|}{ 90th percentile } \\
\hline & cutoff, $\mathrm{ng} / \mathrm{ml}$ & OR & $95 \%$ CI & cutoff, $\mathrm{ng} / \mathrm{ml}$ & OR & $95 \%$ CI & cutoff, $\mathrm{ng} / \mathrm{ml}$ & OR & $95 \% \mathrm{CI}$ \\
\hline MIF & 9.2 & $3.5^{*}$ & $1.8-6.7$ & 10.3 & $3.3^{*}$ & $1.6-6.9$ & 12.6 & $2.8^{*}$ & $1.2-6.5$ \\
\hline TNF & 4.9 & $2.4^{*}$ & $1.3-4.7$ & 5.5 & $3.2^{*}$ & $1.5-6.7$ & 6.0 & $3.1^{*}$ & $1.4-7.2$ \\
\hline IL-6 & 3.1 & 1.5 & $0.7-2.9$ & 4.1 & $2.3^{*}$ & $1.1-5.0$ & 5.7 & $2.5^{*}$ & $1.3-4.8$ \\
\hline Il-10 & 0.655 & $2.3^{*}$ & $1.2-4.5$ & 1.925 & $3.0^{*}$ & $1.4-6.3$ & 3.816 & $4.0^{*}$ & $1.8-9.1$ \\
\hline $\mathrm{CRH}$ bound & 62,000 & $2.4^{*}$ & $1.2-4.6$ & 124,800 & $2.7^{*}$ & $1.3-5.7$ & 189,000 & 2.3 & $1.0-5.5$ \\
\hline CRH total & 63,000 & $3.2^{*}$ & $1.7-6.2$ & 140,400 & $2.7^{*}$ & $1.3-5.7$ & 227,800 & 2.1 & $0.9-5.1$ \\
\hline CRP & $5,667.4$ & $1.9^{*}$ & $1.0-3.7$ & $7,424.1$ & $2.3^{*}$ & $1.1-4.8$ & $9,384.6$ & $2.3^{*}$ & $1.0-5.5$ \\
\hline Cortisol & $1,810.0$ & 1.2 & $0.6-2.3$ & $2,015.6$ & $2.1^{*}$ & $1.0-4.5$ & $2,369.6$ & $2.3^{*}$ & $1.0-5.5$ \\
\hline
\end{tabular}

Cutoffs based on control (term delivery) mothers. OR = Odds ratio; 95\% CI = 95\% confidence intervals. * Significant values.

Table 3. Top ranked models predicting preterm delivery based on biomarker cut-offs

\begin{tabular}{lll}
\hline Cutoff for biomarkers $^{1}$ & & \\
\hline 75th percentile & 85th percentile & 90th percentile \\
\hline Risk factor in model & & \\
MIF & MIF & MIF \\
CRH-total & CRH-free ${ }^{1}$ & CRH-free \\
CRP & CRP & CRP \\
Risk-taking behavior & Risk-taking behavior & Risk-taking behavior \\
Low education attainment & Low education attainment & Low education attainment \\
Risk behavior $\times$ CRP (interaction) & Standing at work ${ }^{2}$ & Previous preterm delivery \\
Area under ROC curve $(95 \%$ CI) & & \\
$0.808 ~(0.743-0.874)$ & $0.780(0.712-0.847)$ & 0.748 (0.677-0.820) \\
\hline${ }^{1}$ CRH-free dichotomized as described in text. ${ }^{2}$ Standing 6 or more hours daily. \\
\hline
\end{tabular}

statistical significance. We found no correlation of MIF with either IL-10 or CRP.

We also examined the correlation of MIF levels with hypothalamic-adrenal-pituitary axis hormones (table 4). There was no correlation between MIF and total CRH levels in either patient group, but there was a strong positive correlation between MIF and free CRH (the level of $\mathrm{CRH}$ that was not bound with $\mathrm{CRH}$-binding protein) in the term deliveries. There was no correlation between $\mathrm{MIF}$ and free $\mathrm{CRH}$ in the women who later delivered preterm. Of note, this analysis is based on a small subset of women with detectable free CRH. There were no statistically significant correlations between MIF and bound $\mathrm{CRH}$ or cortisol.

Multifactor Bio-Demographic Model of Preterm Birth

\section{Discussion}

We found that high levels of MIF, TNF and IL-10 correspond to subsequent $\mathrm{PTB}$ as individual biomarkers, though MIF showed the strongest association with PTB at 2 of the 3 cutoff levels tested. To further assess risk stratification for PTB, we constructed predictive models that bring together putative serum biomarkers of PTB and obstetric history (e.g. previous PTB), sociodemographic factors (e.g. maternal education, standing at work), maternal health behaviors (e.g. tobacco smoking, risk-taking behavior) and perceived psychological stress. In our best performing models, predictors of PTB at all cutoff levels tested were MIF, CRP, risk-taking behavior, and low educational attainment of the mother.

Gynecol Obstet Invest 2010;70:40-46 
Table 4. Correlation of MIF with other putative biomarkers of preterm delivery

\begin{tabular}{lccllrrrl}
\hline \multirow{2}{*}{ Biomarker } & \multicolumn{3}{c}{ Preterm group } & & \multicolumn{2}{c}{ Term group } \\
\cline { 2 - 4 } \cline { 7 - 8 } & $\mathrm{n}$ & $\mathrm{r}_{s}$ & $\mathrm{p}$ & & $\mathrm{n}$ & $\mathrm{r}_{s}$ & $\mathrm{p}$ \\
\hline TNF & 59 & 0.215 & 0.102 & & 121 & 0.221 & $0.015^{*}$ \\
IL-6 & 59 & 0.029 & 0.83 & & 120 & 0.126 & 0.171 \\
IL-10 & 38 & 0.048 & 0.775 & & 49 & -0.044 & 0.763 \\
CRP & 60 & -0.196 & 0.133 & & 121 & 0.058 & 0.53 \\
CRH total & 38 & 0.037 & 0.823 & & 58 & -0.028 & 0.834 \\
CRH bound & 36 & 0.032 & 0.851 & & 57 & -0.128 & 0.341 \\
CRH free & 10 & 0.03 & 0.934 & & 11 & 0.755 & $0.007^{*}$ \\
Cortisol & 60 & 0.094 & 0.477 & & 122 & -0.03 & 0.739 \\
\hline
\end{tabular}

Levels below detection limit for the assays were common for IL-10 and CRH indices, and were coded as missing. $\mathrm{n}=$ Number of subjects; $r_{s}=$ Spearman's rank correlation coefficient.

* Significant correlations $(\mathrm{p}<0.05)$.

Despite general agreement that prematurity is multifactorial, relatively few other studies have reported statistical assessments that combine data on biochemical, microbial, sociodemographic, and behavioral risk factors into a predictive model that can be used in asymptomatic women early in pregnancy [1, 16-19].

That MIF was consistently in our best predictive models (among all the models generated from 22 putative PTB predictor variables from the above categories as well as interaction terms) is strong evidence of its importance in risk assessment of PTB. We reported previously that maternal serum MIF was elevated in women who later delivered preterm [10].

The role of MIF in specific etiologic pathways deserves further consideration. A prior study (using biological samples collected closer to the time of delivery than in the current study) found that high levels of MIF in amniotic fluid were associated with a shorter amniocentesis-to-delivery interval, which implies that intrauterine MIF production could stimulate parturition cascades, particularly during infection [13]. Cortisol normally suppresses TNF, and since MIF overrides this activity of cortisol, we might expect high MIF would correspond to higher TNF. Indeed, MIF and TNF levels were correlated, and both were linked to PTB. Other studies have also found a connection between high serum TNF and PTB $[8,9]$.

The interplay of MIF, cortisol, and CRH is complex, but this triad of molecules is well positioned to form a regulatory loop functioning in pregnancy and parturition $[11,12]$. For example, because CRH can drive MIF

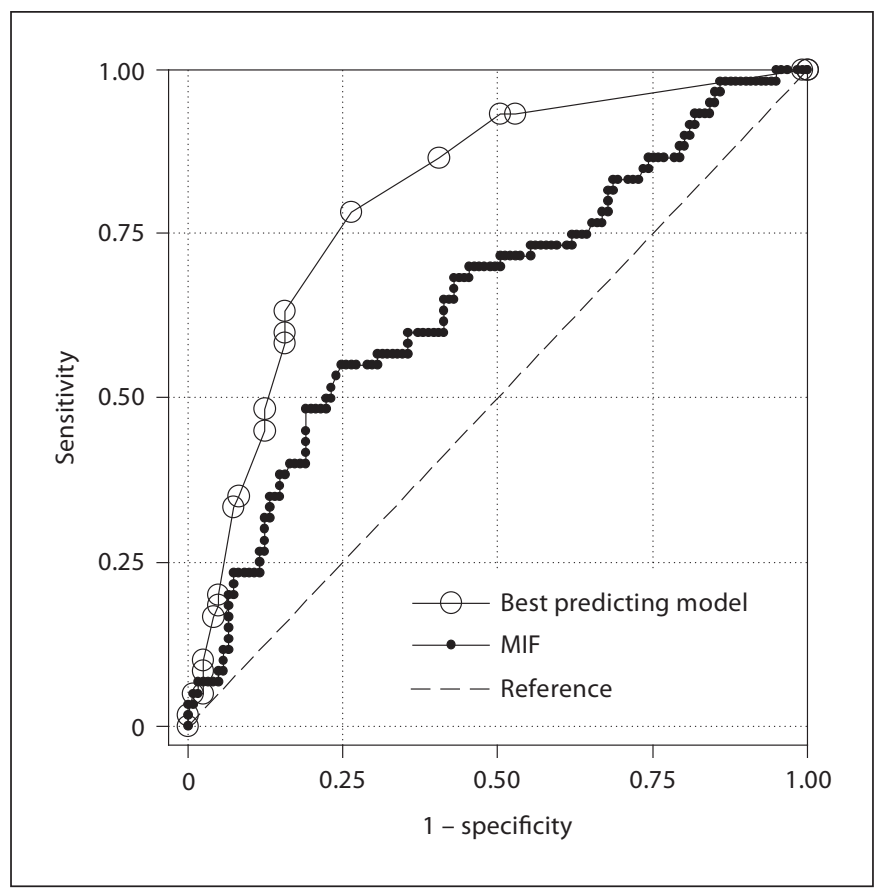

Fig. 1. ROC curve for PTD outcome using 75th percentile cutoff. MIF was the best predictor as an individual biomarker at this cutoff (area under the ROC curve 0.655, 95\% CI 0.569-0.741), but the comprehensive model with six terms performed better (area under the ROC curve $0.808,95 \%$ CI $0.74-0.874$ ).

secretion in other systems (i.e. pituitary), and we observed a positive correlation between free CRH and MIF in the control maternal serum, these immuno-hormonal molecules may be normally coupled at this early to mid stage of pregnancy. Conversely, the lack of such correlation in the women who ultimately delivered preterm may suggest these molecules are pathologically uncoupled many months prior to a preterm delivery. In our assays free $\mathrm{CRH}$ was detectable in only a small subset of women, yet it contributed to the prediction of PTB in our multifactor models.

As reported previously in this population and others, elevations in CRP are statistically associated with PTB despite the limited prognostic value as a single predictor $[4,6]$. The current finding that CRP was among the 6 predictors retained in our multi-factor model suggests that CRP may improve risk stratification when combined with other markers or risk factors. It is possible that MIF, TNF and CRP are identifying the same subpopulation of women with an inflammatory etiology of preterm delivery. Our data, however, argue against this because MIF and CRP were both retained in the comprehensive mod- 
el and were not correlated, which suggest these markers yield complementary information in identifying women at risk of PTB.

High IL-10 was a statistically significant predictor of future PTB at all 3 cutoff levels tested, though this cytokine was detectable in only about half of the serum samples and it was not among the risk factors in our more comprehensive model. As an immunosuppressive cytokine, IL-10 attenuates production of proinflammatory cytokines and has been theorized as an anti-parturition mediator [7]. However, in concert with our findings, Apuzzio et al. [7] found elevated IL-10 in second trimester amniotic fluid of women who later delivered preterm.

There are numerous intermediate or co-varying factors that could plausibly account for the connection between risky behavior and PTB (e.g. substance abuse, higher exposure to sexually transmitted diseases, inadequate self-care or utilization of prenatal services, more unplanned pregnancies). There is also evidence that MIF is a neurobehavioral mediator that can may influence dopaminergic circuits in the brain, which are in turn involved in risk-taking behavior [20,21]. We do not have sufficient data to determine if such neural properties of MIF are relevant to PTB, or whether the relationship between MIF and PTB is mainly being played out in the periphery.

Our comprehensive models compare favorably with other models in the literature. Goodwin et al. [22] reported another complex multifactor model emphasizing the importance of 7 maternal demographic factors (including maternal education) in PTB prediction; their model yielded an area under the ROC curve of 0.75 (compared to 0.81 for our best multifactor model). The model reported by Misra et al. [3], which included biomedical and psychosocial factors but not serum biomarkers, did not find maternal education to be a predictor of PTB. Two additional studies that used maternal characteristics but not biomarkers in their models calculated areas under the ROC curve of $0.70-0.73[23,24]$. The multivariate model reported by Smith et al. [17], which took into account two biomarkers ( $\alpha$-fetoprotein and human chorionic gonadotropin) and maternal sociodemographic characteristics (but not specifically maternal education) yielded an area under the ROC curve of 0.62 for prediction of delivery at 33-36 weeks.

Our results have to be confirmed and refined in other larger samples. Clinicians often make assessments and judgments based on an overall synthesis of data. There are clearly difficult clinical scenarios where the right course of action is not obvious. With increasing use of bedside computing, multivariate models hold some promise for implementation as a means to direct attention to the women at highest risk for PTB. There is a major need to improve the treatment of PTB, and we contend that the science guiding PTB prediction should be mutually informative with advances in treatment. Etiologically, our models point to behavioral risk factors in addition to biological markers. This raises the prospect of lowering rates of PTB by behavioral interventions.

There are several limitations to this study. Our findings from a population of mostly healthy white women may not generalize to more mixed ethnic populations with less access to healthcare, greater psychosocial stress levels, and higher rates of PTB [25]. The relationships observed among putative molecular mediators are purely correlational, and cannot prove a specific causal mechanism or bimolecular interaction. Our assays were not sufficiently sensitive to detect low levels of CRH or IL-10. Some loss of immunoreactivity may have occurred during storage of serum samples, particularly for IL-10 [26]. Still, we had sufficient samples with measurable IL-10 values to find an association between IL-10 and subsequent PTB.

The strong correlation of free $\mathrm{CRH}$ with MIF was based on relatively few detectable samples and probably represents women with $\mathrm{CRH}$ levels that are on the upper end of the spectrum. The statistical approach used to select the 'best' predictive models was exploratory. Moreover, as the ROC areas were estimated from the same data as the models were derived, they are biased upwards with respect to the general population.

In conclusion, by modeling molecular indices together with other risk factors discernable at an early prenatal visit, we show a method that helps optimize risk stratification for PTB.

\section{Acknowledgements}

We thank Dr. Denise Raynor and Dr. Kristina Weis for assistance in the early conception of this project. We thank Sicily Garvin for her expert technical work, and Sandeep Pathi for his assistance with samples and assays. This study was supported by a National Institute of Mental Health grant, 5 R21 MH068513-3 (to B.D.P.), a grant from the March of Dimes Foundation, New York (PERI-98), and a grant from NARSAD (B.D.P.).

Gynecol Obstet Invest 2010;70:40-46 


\section{References}

1 Erickson K, Thorsen P, Chrousos G, Grigoriadis DE, Khongsaly O, McGregor J, Schulkin J: Preterm birth: associated neuroendocrine, medical, and behavioral risk factors. J Clin Endocrinol Metab 2001;86:2544-2552.

2 Goldenberg RL, Culhane JF, Iams JD, Romero R: Epidemiology and causes of preterm birth. Lancet 2008;371:75-84.

3 Misra DP, O’Campo P, Strobino D: Testing a sociomedical model for preterm delivery. Paediatr Perinat Epidemiol 2001;15:110122.

$\checkmark 4$ Hvilsom GB, Thorsen P, Jeune B, Bakketeig LS: C-reactive protein: a serological marker for preterm delivery? Acta Obstet Gynecol Scand 2002;81:424-429.

5 Pitiphat W, Gillman MW, Joshipura KJ, Williams PL, Douglass CW, Rich-Edwards JW: Plasma C-reactive protein in early pregnancy and preterm delivery. Am J Epidemiol 2005;162:1108-1113.

-6 Lohsoonthorn V, Qiu C, Williams MA: Maternal serum C-reactive protein concentrations in early pregnancy and subsequent risk of preterm delivery. Clin Biochem 2007;40: 330-335.

-7 Apuzzio J, Chan Y, Al-Khan A, Illsley N, Kim PL, Vonhaggen S: Second-trimester amniotic fluid interleukin-10 concentration predicts preterm delivery. J Matern Fetal Neonatal Med 2004;15:313-317.

>8 Gücer F, Balkanli-Kaplan P, Yüksel M, Yüce MA, Türe M, Yardim T: Maternal serum tumor necrosis factor-alpha in patients with preterm labor. J Reprod Med 2001;46:232236.

-9 Vitoratos N, Papadias K, Makrakis E, Christodoulakos G, Panoulis K, Creatsas G: Association between serum tumor necrosis factor-alpha and corticotropin-releasing hormone levels in women with preterm labor. J Obstet Gynaecol Res 2006;32:497501.
10 Pearce BD, Garvin SE, Grove J, Bonney EA, Dudley DJ, Schendel DE, Thorsen P: Serum macrophage migration inhibitory factor in the prediction of preterm delivery. Am J Obstet Gynecol 2008;199:46.e1-e6.

-11 Vigano P, Cintorino M, Schatz F, Lockwood CJ, Arcuri F: The role of macrophage migration inhibitory factor in maintaining the immune privilege at the fetal-maternal in terface. Semin Immunopathol 2007;29:135150.

12 Flaster H, Bernhagen J, Calandra T, Bucala $\mathrm{R}$ : The macrophage migration inhibitory factor-glucocorticoid dyad: regulation of inflammation and immunity. Mol Endocrinol 2007;21:1267-1280.

13 Chaiworapongsa T, Romero R, Espinoza J, Kim YM, Edwin S, Bujold E, Gomez R, Kuivaniemi $\mathrm{H}$ : Macrophage migration inhibitory factor in patients with preterm parturition and microbial invasion of the amniotic cavity. J Matern Fetal Neonatal Med 2005; 18 : 405-416.

14 Bacher M, Meinhardt A, Lan HY, Dhabar FS Mu W, Metz CN, Chesney JA, Gemsa D, Donnelly T, Atkins RC, Bucala R: MIF expression in the rat brain: Implications for neuronal function. Mol Med 1998;4:217230.

15 Ramsay JE, Ferrell WR, Crawford L, Wallace AM, Greer IA, Sattar N: Maternal obesity is associated with dysregulation of metabolic, vascular, and inflammatory pathways. J Clin Endocrinol Metab 2002;87:4231-4237.

16 Ruiz RJ, Fullerton J, Brown CE, Dudley DJ: Predicting risk of preterm birth: the roles of stress, clinical risk factors, and corticotropin-releasing hormone. Biol Res Nurs 2002; 4:54-64.

17 Smith GC, Shah I, White IR, Pell JP, Crossley JA, Dobbie R: Maternal and biochemical predictors of spontaneous preterm birth among nulliparous women: a systematic analysis in relation to the degree of prematurity. Int Epidemiol 2006;35:1169-1177.

$\checkmark 18$ Meis PJ, Michielutte R, Peters TJ, Wells HB, Sands RE, Coles EC, Johns KA: Factors associated with preterm birth in Cardiff, Wales. II. Indicated and spontaneous preterm birth. Am J Obstet Gynecol 1995;173 597-602.
19 Hendler I, Goldenberg RL, Mercer BM, Iams JD, Meis PJ, Moawad AH, MacPherson CA, Caritis SN, Miodovnik M, Menard KM, Thurnau GR, Sorokin Y: The preterm prediction study: association between maternal body mass index and spontaneous and indicated preterm birth. Am J Obstet Gynecol 2005; 192:882-886.

20 Matsunaga J, Sinha D, Pannell L, Santis C, Solano F, Wistow GJ, Hearing VJ: Enzyme activity of macrophage migration inhibitory factor toward oxidized catecholamines. J Biol Chem 1999;274:3268-3271.

-21 Weingarten P, Zhou QY: Protection of intracellular dopamine cytotoxicity by dopamine disposition and metabolism factors. J Neurochem 2001;77:776-785.

22 Goodwin LK, Iannacchione MA, Hammond WE, Crockett P, Maher S, Schlitz K: Data mining methods find demographic predictors of preterm birth. Nurs Res 2001;50:340345.

$>23$ Eastaugh JL, Smye SW, Snowden S, Walker JJ, Dear PRF, Farrin A: Comparison of neural networks and statistical models to predict gestational age at birth. Neural Comput Applic 1997;6:158-164.

24 Tan H, Wen SW, Chen XK, Demissie K, Walker M: Early prediction of preterm birth for singleton, twin, and triplet pregnancies. Eur J Obstet Gynecol Reprod Biol 2007;131: 132-137.

25 Hogue CJ, Bremner JD: Stress model for research into preterm delivery among black women. Am J Obstet Gynecol 2005;192:S47S55.

-26 Kenis G, Teunissen C, De Jongh R, Bosmans E, Steinbusch H, Maes M: Stability of interleukin 6, soluble interleukin 6 receptor, interleukin 10 and CC16 in human serum. Cytokine 2002;19:228-235 